\title{
Uso e cobertura do solo da bacia hidrográfica do Ribeirão Salabra, MS
}

\author{
Dulce Buchala Bicca Rodrigues ${ }^{1}$ \\ Paulo Tarso Sanches de Oliveira ${ }^{1}$ \\ Teodorico Alves Sobrinho ${ }^{1}$ \\ Elói Panachuki ${ }^{2}$ \\ ${ }^{1}$ Universidade Federal de Mato Grosso do Sul - UFMS \\ Caixa Postal 549 - 79070-900 - Campo Grande - MS, Brasil \\ paulotarsoms@hotmail.com; dulcebbr@gmail.com; talves@ufms.br \\ ${ }^{2}$ Universidade Estadual de Mato Grosso do Sul - UEMS, \\ Caixa Postal -351 - 79804-970 - Dourados - MS, Brasil \\ eloip@uems.br
}

\begin{abstract}
The survey of the use and land cover is important for implementation of agricultural and environmental planning. Thus, this study was to perform the mapping and quantification of current land use in the catchment area of Ribeirão Salobra, MS, aiming to provide support for environmental and agricultural planning. The study area is located in Terenos City, MS, in the vicinity of the Pantanal biome. Images of sensor Thematic Mapper (TM) of Landsat 5 were processed in software SPRING. For this, the processes of geometric correction, segmentation, region extraction, classification, and mapping were achieved, and field work to validate the use classes identified. In the thematic map generated, the classes cerrado, savanna, degraded pasture and pasture have covered approximately $13 \%, 10 \%, 35 \%$ and $38 \%$ of the area, respectively. The predominance of grassland, about $73 \%$, used in livestock farming, was verified. The methodology shows to be satisfactory and capable of supporting the agricultural and environmental planning. Moreover, the gratuity and easy access to data provide further new studies in the basin.
\end{abstract}

Palavras-chave: geoprocessing, agricultural and environmental planning, degradation of soil, geoprocessamento, planejamento agrícola e ambiental, degradação do solo.

\section{Introdução}

Um dos principais problemas de degradação ambiental são os processos de erosão hídrica, capazes de degradar o solo e a água. Nearing et al. (2005) e Vente et al. (2008) relatam que atualmente esses processos vêm sendo acelerados pelo inadequado uso do solo e em virtude de mudanças climáticas. Para Simões e Coiado (2003) o homem tem sido o mais ativo agente ligado à destruição do solo, seja por meio de práticas agrícolas inadequadas, seja através da implantação de loteamentos sem considerar a fragilidade do terreno. Assim, verifica-se a intensificação de fenômenos que proporcionam degradação ambiental, como a erosão hídrica. Segundo Bueno e Stein (2004), a dinâmica natural da paisagem por meio dos processos naturais, juntamente com a quebra no equilíbrio naturalmente estabelecido, provocada pela ação do homem, determina a intensidade dos processos erosivos.

De acordo com Pandey et al. (2007), a prioridade deve ser dada para a conservação dos recursos do solo promovendo sua utilização de maneira adequada. Assim, para alcançar tal objetivo o planejamento, a conservação e o gerenciamento de bacias hidrográficas são vitais. Déstro e Campos (2006) descrevem o levantamento do uso do solo como etapa primordial para qualquer ação de planejamento territorial e recursos naturais. Neste sentido, vem sendo utilizado com êxito geotecnologias, tais como sensoriamento remoto e Sistemas de Informação Geográfica (SIG), pois, apresentam confiabilidade e baixa relação custo e beneficio. 
De acordo com Florenzano (2002), as imagens obtidas por sensoriamento remoto contribuem na identificação de diferentes usos do espaço terrestre. $\mathrm{O}$ aspecto multitemporal dessas imagens permite acompanhar as transformações do espaço ao longo do tempo. $\mathrm{O}$ avanço da tecnologia de novos softwares, que são utilizados para processar imagens de satélites, viabilizou e intensificou o uso do sensoriamento remoto para diversas finalidades. Assim, diversos estudos vêm sendo realizados utilizando tais técnicas, na tentativa de melhor entender a dinâmica de exploração dos recursos ambientais (Campos et al., 2004; Ribeiro e Campos, 2007; Bonnet et al., 2008; Beskow et al., 2009).

Deste modo, este trabalho teve por objetivo realizar o mapeamento e a quantificação do atual uso do solo da bacia hidrográfica do Ribeirão Salobra, MS, com intuito de fornecer subsídios para estudos de planejamento agrícola e ambiental da bacia hidrográfica.

\section{Materiais e Métodos}

\section{1 Área de estudo}

O estudo foi realizado tomando-se por base a bacia hidrográfica do Ribeirão Salobra, localizada à $20^{\circ} 19^{\prime} \mathrm{S}$ de latitude e $55^{\circ} 15^{\prime} \mathrm{W}$ de longitude, com área aproximada de 540 $\mathrm{km}^{2}$. É pertencente ao município de Teremos, distante cerca de $50 \mathrm{~km}$ de Campo Grande, MS.

A bacia em estudo faz parte da sub-bacia do Rio Miranda pertencente à Bacia do Alto Paraguai (BAP). Possui características de um relevo de transição entre a escarpa da Serra de Maracaju e a depressão Pantaneira (Figura 1).

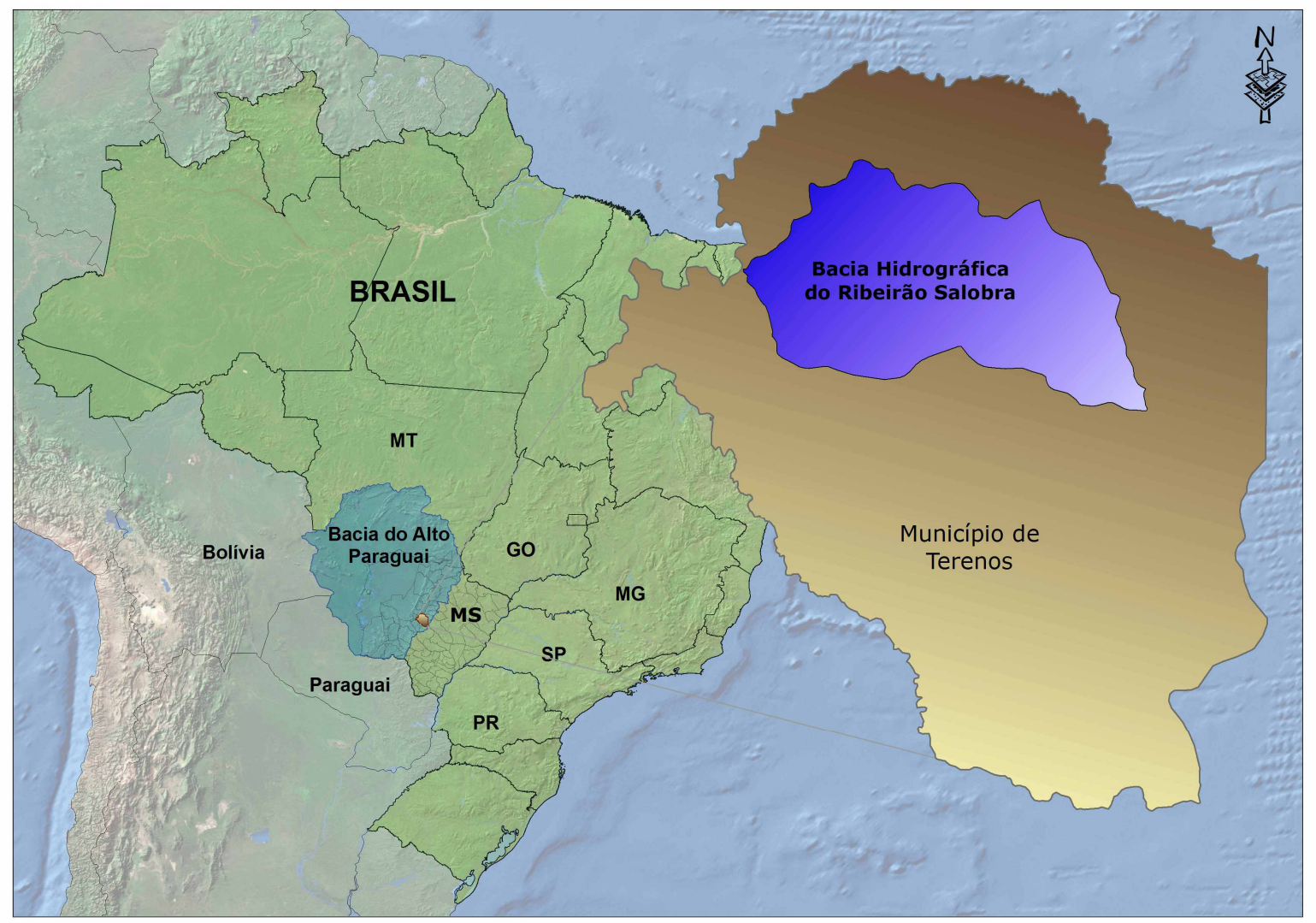

Figura 1. Localização da área em estudo

\subsection{Leitura e registro da imagem}

Utilizando o software SPRING 4.3.3 (Câmara et al., 1996), foi desenvolvido um banco de dados tomando como base o elipsóide de referência Córrego Alegre, Meridiano Central $57^{\circ}$ W Gr., Fuso 21 Sul e sistema de coordenadas em Universal Transversa de Mercator (UTM). 
A base cartográfica de referência utilizada neste trabalho foi às cartas topográficas folhas Campo Grande SF. 21-X-B-II (DSG, 1979) e Palmeiras SF. 21-X-B-I (DSG, 1972), ambas na escala 1:100.000. Primeiramente, estas cartas foram digitalizatas com resolução de 300 dpi e convertidas para o formato GRIB no módulo IMPIMA do SPRING. Posteriormente, procedeu-se o registro utilizando-se polinômio de $2^{\circ}$ grau e o interpolador vizinho mais próximo. As imagens foram registradas com 20 pontos de controle, obtendo-se valores de erro inferiores a 0,5 pixel.

A obtenção dos dados de uso e cobertura do solo foi realizada pelo mapeamento obtido a partir da classificação da imagem digital do sensor Thematic Mapper (TM) do satélite LANDSAT 5, com data de passagem de 2008, referente a órbita/ponto 225/74 (INPE, 2008). Esta imagem foi convertida para o formato GRIB no módulo IMPIMA do SPRING e procedeu-se a etapa de correção geométrica, na qual, foi utilizado polinômio de $2^{\circ}$ grau e o interpolador vizinho mais próximo, tendo-se por base de referência as cartas topográficas já georreferenciadas. A imagem foi registrada com 30 pontos de controle, obtendo valor de erro inferior a 0,6 pixel, o equivalente a um erro menor que 18 metros. Após o registro, realizou-se o procedimento de realce de contraste (ajuste linear) com o objetivo de facilitar a visualização e interpretação da imagem.

\subsection{Segmentação e classificação da imagem}

No processo de classificação da imagem realizou-se o procedimento de segmentação e classificação supervisionada por regiões, disponível no SPRING 4.3.3. De acordo com Moreira (2005) e Novo (2008), os classificadores por regiões utilizam como critério de decisão a informação espectral de cada pixel da imagem e a informação espacial que envolve a relação entre o pixel e seus vizinhos. Estes classificadores procuram simular o comportamento de um fotointérprete ao reconhecer áreas homogêneas nos dados orbitais, baseados nas propriedades espectrais e espaciais dessas áreas na imagem. $\mathrm{O}$ processo de classificação realizado neste estudo pode ser dividido nas seguintes etapas: segmentação; extração de regiões; classificação; e, mapeamento.

Na realização da segmentação por processo de crescimento por regiões é necessária a definição do valor de dois parâmetros, o limiar de similaridade e o de área mínima. Segundo Novo (2008), o limiar de similaridade representa a menor diferença aceita entre o valor médio de dois pixels ou conjuntos de pixels, para que sejam alocados a segmentos distintos. Já o limiar de área mínima, representa o tamanho mínimo do segmento definido pelo analista. De acordo com Coutinho (1997), os valores ótimos a serem fixados para a segmentação de imagens, dependem principalmente dos padrões de repartição espacial dos objetos de cada área e da definição da generalização cartográfica ideal, em função da escala cartográfica da abordagem realizada. Não existe, portanto, uma padronização de valores destas variáveis para a obtenção de bons resultados.

Realizou-se a segmentação da imagem, utilizando as bandas 3, 4 e 5 do LANDSAT 5 TM, testando diversos valores de similaridade e área, sendo adotado os valores de similaridade 20 e área 40 (Figura 2). Depois da escolha dos critérios foi realizada a segmentação, aquisição e amostras de treinamento e classificação. No entanto, antes da fase de treinamento, foram definidas, as classes de uso e ocupação do solo por meio da interpretação visual da imagem.

A partir das imagens segmentadas realizou-se a extração de atributos das regiões e a classificação utilizando-se o classificador Bhattachaarrya com limiar de aceitação de $90 \%$. As classes geradas neste processo foram trabalhadas na etapa de mapeamento e edição matricial. 


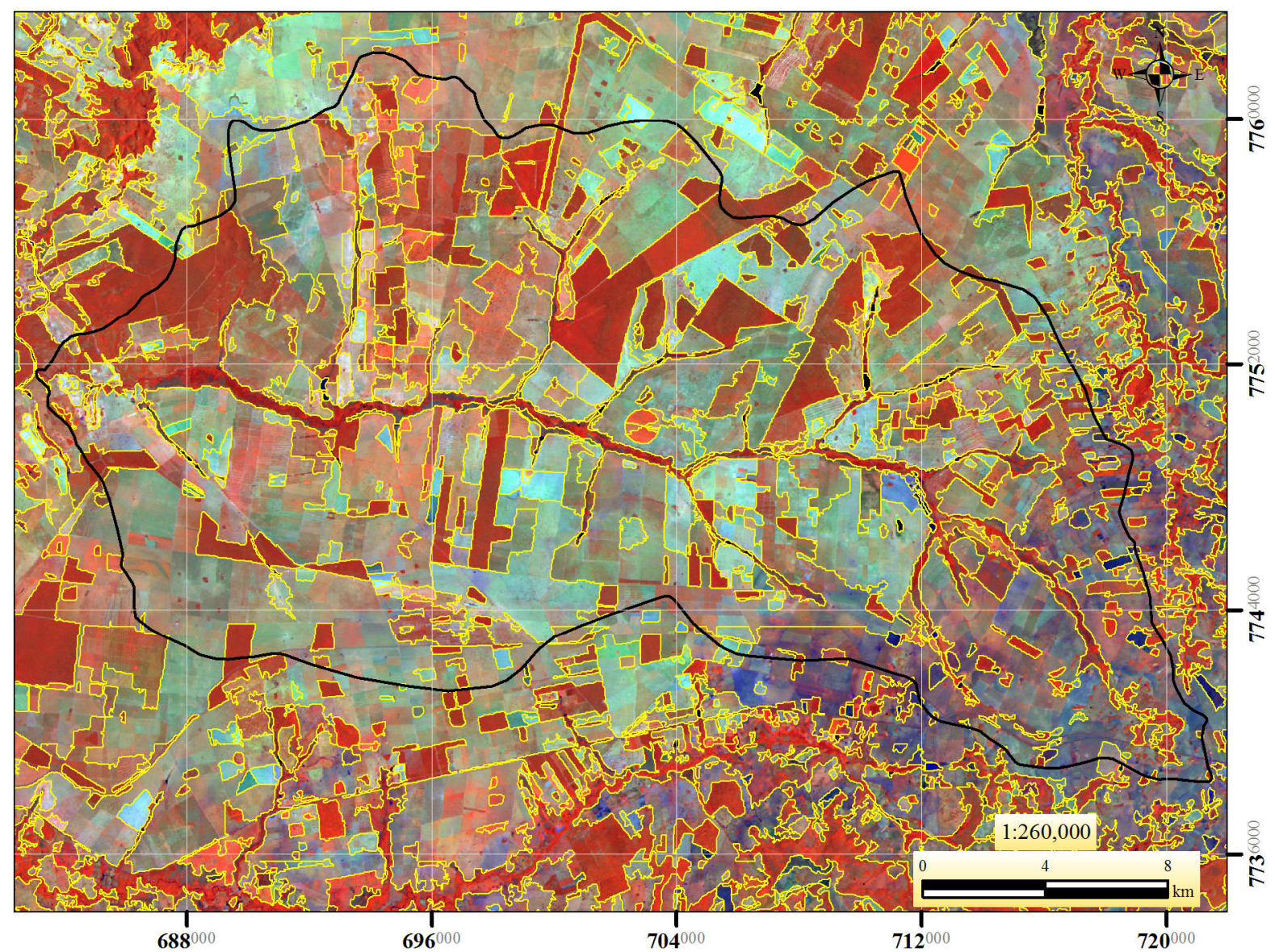

Figura 2. Imagem LANDSAT 5 TM (4R 5G 3B) segmentada

$O$ resultado da classificação foi comparado às imagens LANDSAT 5 TM, composição 4R 5G 3B e, a partir desta e de trabalhos de campo, realizou-se a edição matricial dos polígonos que não foram classificados adequadamente. Posteriormente, procedeu-se a conversão raster para vetor no SPRING e quantificou-se as áreas classificadas.

\section{Resultados e discussão}

Na Tabela 1 encontra-se representado as classes de uso do solo verificadas na bacia hidrográfica do Ribeirão Salobra. Nota-se predominância de áreas de pastagens (aproximadamente $73 \%$ ) ocupadas por pecuária extensiva.

Tabela 1. Classes de uso do solo

\begin{tabular}{ccc}
\hline \multirow{2}{*}{ Classes de uso e ocupação do solo } & \multicolumn{2}{c}{ Área } \\
\cline { 2 - 3 } & $\mathbf{k m}^{\mathbf{2}}$ & $\mathbf{\%}$ \\
\hline Cerradão & 71 & 13.12 \\
Cerrado & 58 & 10.73 \\
Pastagem degradada & 189 & 34.93 \\
Pastagem & 207 & 38.27 \\
Água & 1 & 0.26 \\
Áreas úmidas & 5 & 0.98 \\
Culturas & 9 & 1.72 \\
\hline
\end{tabular}

A classe culturas é composta por silvicultura e citricultura, e encontram-se na parte sul da bacia hidrográfica (Figura 3). 


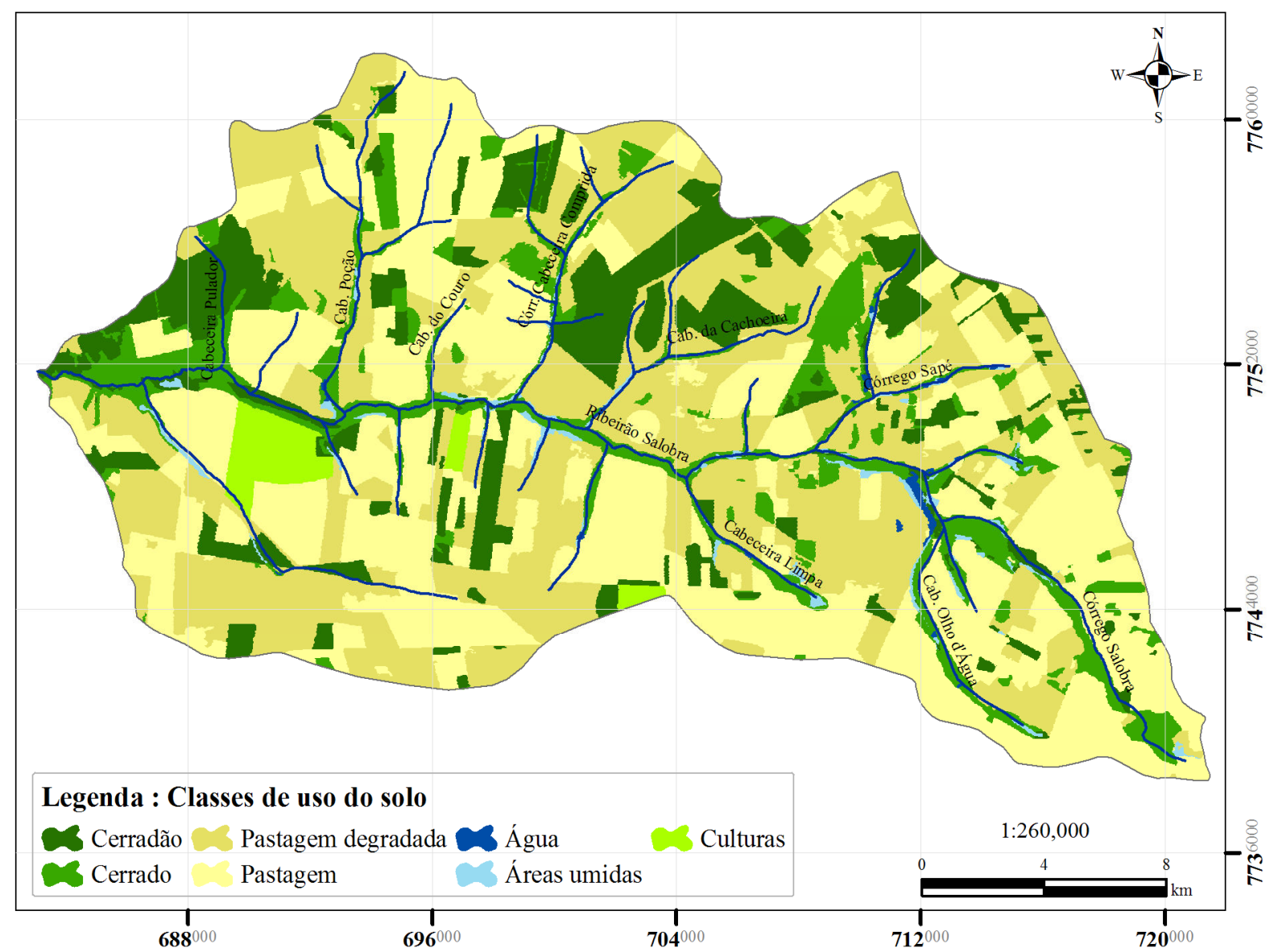

Figura 3. Classes de uso do solo

Verificou-se de forma qualitativa que não vem sendo respeitada a legislação quanto às Áreas de Preservação Permanente (APP), Resolução CONAMA nº 303/2002 (BRASIL, 2002) e de Reserva Legal, Lei $n^{\circ}$ 4.771/1965 (BRASIL, 1965), assim, áreas de vegetação nativa estão dando lugar principalmente a pastagens. Observou-se também a construção de barragens em boa parte das nascentes do Ribeirão Salobra. De acordo com Smaniotto et al. (2003), a extensão territorial do estado de Mato Grosso do Sul, sua estrutura fundiária, dificuldade de acesso e a falta de condições humanas e operacionais, comuns na realidade nacional, não tem permitido uma atuação eficaz no gerenciamento das APPs e de Reserva Legal obrigatórias nas propriedades rurais.

\section{Conclusões}

No mapa temático gerado, as classes cerradão, cerrado, pastagem degradada e pastagem contemplaram, aproximadamente, $13 \%, 10 \%, 35 \%$ e $38 \%$ da área, respectivamente. Podendose verificar predomínio de pastagens, cerca de $73 \%$, utilizadas na pecuária extensiva.

A metodologia utilizada mostra-se satisfatória e capaz de subsidiar o planejamento agrícola e ambiental da bacia hidrográfica em estudo.

A gratuidade e facilidade de acesso aos dados proporcionam a continuação de novos estudos na bacia hidrográfica.

\section{Agradecimentos}

Os autores expressam seus agradecimentos ao CNPq e a FUNDECT, pelo auxílio financeiro concedido para a realização do trabalho. 


\section{Referências bibliográficas}

Beskow, S.; Mello, C.R.; Norton, L.D.; Curi, N.; Viola, M.R.; Avanzi, J.C. Soil erosion prediction in the Grande River Basin, Brazil using distributed modeling. Catena, v.79, p.49-59, 2009.

Bonnet, B. R. P.; Ferreira, L. G.; Lobo, F. C. Relações entre qualidade da água e uso do solo em Goiás: uma análise à escala da bacia hidrográfica. Revista Árvore, v.32, n.2, p.311-322, 2008.

BRASIL. Conselho Nacional de Meio Ambiente. Resolução CONAMA no 303. Dispõe sobre parâmetros, definições e limites de Áreas de Preservação Permanente. Brasília: 2002. Diário Oficial da União, 20 de março de 2002.

BRASIL. Lei no 4.771. Institui o novo Código Florestal. Brasília: 1965. Diário Oficial da União, 15 de dezembro 1965.

Bueno, C. R. P.; Stein, D. P. Potencial natural e antrópico de erosão na região de Botas, Estado de São Paulo. Acta Scientiarum Agronomy. v. 26, n. 1. p. 1-5. 2004.

Câmara, G.; Souza, R.C.M.; Freitas, U.M.; Garrido, J.; IR, F.M. SPRING: Integrating remote sensing and GIS by object-oriented data modelling. Computers \& Graphics, v. 20, n. 3, p. 395-403, 1996.

Campos, S.; Araújo Junior, A. A. Barros, Z. X.; Cardoso, L. G.; Piroli, E. L. Sensoriamento remoto e geoprocessamento aplicados ao uso da terra em microbacias hidrográficas, Botucatu - SP. Eng. Agríc., v.24, n.2, p.431-435, 2004.

Coutinho, A. M. Segmentação e classificação de imagens Landsat - TM para o mapeamento dos usos da terra na região de Campinas, SP. São Paulo, 1997. 139p. Dissertação (Mestrado em Ciências). Universidade de São Paulo.

Déstro, G. F. G.; Campos, S. SIG-SPRING na caracterização do uso dos solos a partir de imagens do satélite CBERS. Energ. Agric.v.21, n.4, p.28-35, 2006.

DSG (Diretoria do Serviço Geográfico). Carta Campo Grande. Folha SF. 21-X-B-II. Escala 1:100.000. Região Centro-Oeste do Brasil. Carta 1979.

DSG (Diretoria do Serviço Geográfico). Carta Sidrolândia. Folha SF. 21-X-B-V. Escala 1:100.000. Região Centro-Oeste do Brasil. Carta 1972.

Florenzano, T. G. Imagens de satélite para estudos ambientais. São Paulo: Oficina de Textos. 97p. 2002.

INPE - Instituto Nacional de Pesquisas Espaciais. Imagem LANDSAT 5. Sensor TM. Canais 1 ,2 ,3 ,4, 5, 6, 7. São José dos Campos: Instituto Nacional de Pesquisas Espaciais. Imagem de Satélite. Órbita 225 Ponto 74 . De 18 de dezembro de 2008. Disponível em http://www.dgi.inpe.br/CDSR/. Acesso em: 17 mar., de 2009.

Moreira, M. A. Fundamentos do sensoriamento remoto e metodologias de aplicação. $3^{\text {a }}$ ed. Viçosa: UFV. 320p. 2005.

Nearing, M. A.; Jetten, V.; Baffaut, C.; Cerdan, O.; Couturier, A.; Hernandez, M.; Le Bissonnais, Y.; Nichols, M. H.; Nunes, J. P.; Renschler, C. S.; Souchère, V.; van Oost, K. Modeling response of soil erosion and runoff to changes in precipitation and cover. Catena, v.61, p.131-154, 2005.

Novo, E. M. L. M. Sensoriamento remoto: princípios e aplicações. São Paulo: Blucher, 363p. 2008.

Pandey, A.; Chowdary, V.M.; Mal, B.C. Identification of critical erosion prone areas in the small agricultural watershed using USLE, GIS and remote sensing. Water Resources Management, n. 21, p. 729-746, 2007.

Ribeiro, F.L.; Campos, S. Vulnerabilidade à erosão do solo da Região do Alto Rio Pardo, Pardinho, SP. Revista Brasileira de Engenharia Agrícola e Ambiental, v.11, n.6, p.628-636, 2007.

Simões, S. J. C.; Coiado, E. M. Processos erosivos. In: Paiva, J. B. D.; Paiva, E. M. C. D. (Org.). Hidrologia aplicada à gestão de pequenas bacias hidrográficas. Porto Alegre: ABRH, p. 283-293. 2003.

Smaniotto, C. R.; Decanini, M. M. S.; Silva, E. A. SIG no licenciamento e físcalização da cobertura vegetal natural do estado de Mato Grosso do Sul. Bol. Ciênc. Geod. v. 9, n.1. p. 39-51. 2003.

Vente, J.; Poesen, J.; Verstraeten, G.; Rompaey, A. V.; Govers, G. Spatially distributed modelling of soil erosion and sediment yield at regional scales in Spain. Global and planetary change, v. 60, p.393-415, 2008. 